ovary of its own side. This confirms, in the living normal marsupial, observations made by Hartmann (loc. cit. p. 441) on pregnant opossums from which the uterus of one side and the ovary of the other had been removed. This supports, too, Hartmann's contention that operative interference had nothing to do with his results. When the egg in the pregnant uterus has reached the stage where it possesses a well-formed primitive streak, involution of the sterile uterus begins to take place, while the other uterus undergoes the usual enlargement associated with normal development.

Seeing that a single corpus luteum, situated in one ovary, influences, up to this stage, the activities of both uteri, is it not reasonable to suppose that, at the time of involution of the sterile uterus, the secretory activity of the corpus luteum has begun to wane? As such seems to be the case, it is obvious that parturition could scarcely be caused by the action of the corpus luteum.

The only other way of accounting for the regressive changes in the sterile uterus is to suggest that they may be due to some inhibitory influence, but it does not seem that any such influence could exist without involving both uteri.

University of Tasmania, May 4.

\section{Personal and Impersonal Styles in Scientific Communications.}

Deliberately encouraged by some editors, though not, I am assured, by the Editor of NATURE, a prejudice is growing against the use of the first person in scientific writings. If it were part of a more general prejudice against the increasing egotism of scientists (manifested, for example, in squabbles over priority and the assignment of credit), it would be admirable, though probably futile. But it is not; it is merely the stylistic fad of the moment, and is wholly contemptible. Truly, it is not well to be always saying ' $I$ '; but there is no virtue, moral or literary, in the mechanical substitution of the third person for the first. The literary vice is not the repetition of pronouns or of any pronoun in particular; it is needless repetition in general; pronouns are mentioned specifically only because it is particularly tempting to repeat them too often. It is very much worse to repeat a cumbrous and artificial phrase than to repeat a pronoun; and when we find our pages, sedulously cleared of 'I's,' besprinkled with ' the present writer' and 'the author of the present paper,' it is surely time to protest against a Pharisaical misinterpretation of the Law.

Pedants are too often ingenious in excusing their sins; and somebody will probably discover literary sanction even for this last abomination, perhaps in the introductions to the Waverley novels. I would therefore insist that, even if Scott's circumstances had been normal, even if he had said 'the present' when he meant 'this,' Scott and his like, for all their excellencies, would still not be fit models for those whose first duty is to be brief and direct. If we must imitate anyone, let it be the masters of our craft; let us try to learn from Faraday, Rayleigh, Huxley, and many others how to say ' $I$ ' freely, naturally, with elegance and with dignity.

For there are real difficulties, peculiar to scientific writing, that it would be foolish to ignore. An analysis of about 200 of the worst flounderings in recent journals suggests that they arise in three ways. First, Prof. Smith wants to refer to his own earlier work, but does not like to say 'I found in 1924 ...' Why should he not denote himself, as he would any other person, by his name, and say, 'Smith found . . ' Second, impersonal narrative becomes tedious; we want to vary 'The next experiment was . . ' with 'Then I tried ...' Why should we not? The pronoun here is quite unobtrusive; for no emphasis can fall on it, as reading aloud will show. Last, we want to make a personal statement, distinguishing our opinion from facts or from the opinions of others. Here a moral issue is raised. If we are not prepared to make a personal statement in personal form, are we justified in making it at all ? Can true modesty, or any other virtue, permit me to occupy valuable space in airing my views and yet forbid me to call them mine ?

Norman R. CAMPBELL.

\section{The Arc Spectrum of Chlorine.}

Regularities in the arc spectrum of $\mathrm{Cl}$ were first discovered by Turner (Phys. Rev., 27, 397; 1926), who found three pairs showing the difference $\mathbf{8 8 0}$ $\mathrm{cm}^{-1}$. A consistent extension of this work into the visible, and theoretical interpretation has, however, never been attempted, especially because the separation of the chlorine lines into arc and spark lines had not been possible. Thus the data given in Kayser's "Handbuch" contain lines of both types.

Recently Mr. K. Asagoe, of this laboratory, has been able to identify 23 arc lines in the region between $4700 \mathrm{~A}$. and $4200 \mathrm{~A}$. The method is similar to that used by Wood and Kimura (Astrophys. Jour., 46, 181; 1917 ) in separating the are and spark lines of iodine. An examination of these Cl lines shows that they form the combination $4 s^{4} P-4 p^{4}(S, P, D)$. The frequency differences between the five lower levels are $530.4 ; 338.7 ; 1399.1 ; 640.8 \mathrm{~cm}^{-1}$. Attention has already been directed to the first of these by de Bruin (Amsterdam Proc., 30, 19 ; 1927). But these are just the differences which one finds between Turner's lines in the far ultra-violet region. They must consequently be identified as ${ }^{2} P\left(3 p^{6}\right)-{ }^{42} P\left(3 p^{5}, 4 s\right)$. In the following table the individual lines, their intensities and levels, are given :

$\begin{array}{llllll}1396 \cdot 5 & 3 & { }^{2} P_{1}-{ }^{4} P_{2} & 1351 \cdot 7 & 3 & { }^{2} P_{2}-{ }^{2} P_{1} \\ 1389 \cdot 9 & 4 & { }^{2} P_{2}-{ }^{4} P_{3} & 1347 \cdot 2 & 5 & { }^{2} P_{2}-{ }^{2} P_{2} \\ 1379 \cdot 6 & 5 & { }^{2} P_{2}-{ }^{4} P_{2} & 1335 \cdot 8 & 2 & { }^{2} P_{2}-{ }^{2} P_{1} \\ 1363.5 & 5 & { }^{2} P_{1}-{ }^{2} P_{2} & & & \end{array}$

\section{All the lines found by Turner are thus accounted} for. Although the terms arising from the configurations $\left(3 p^{6}\right)$ and $\left(3 p^{5}, 4 s\right)$ are still approximately ' normal,' that is, the corresponding vector coupling is of Russell-Saunders type, the higher term group arising from $\left(3 p^{5}, 4 p\right)$, which the visible lines have as initial state, has a decidedly different character; this term group is-as one usually puts it- of "higher rank.' The situation is quite similar to that in the arc spectrum of argon, where, according to the classification of Meissner (Zeit f. Phys., 39, 172; 1926), the four $4 s$ levels arise from a Russell-Saunders coupling, whereas the ten $4 p$ levels which are grouped quite irregularly, are apparently due to some intermediate coupling (Goudsmit-Uhlenbeck). There is consequently a certain ambiguity in assigning $l$-values to the $\left(3 p^{5}, 4 p\right)$ group. However, the weakness of intercombinations leads to a distinction between doublet and quartet levels.

Corresponding results in bromine and iodine have been obtained, but the work is still in progress.

My thanks are due to Prof. M. Kimura, through whose kindness my stay in Kyoto has been possible.

Physics Department, Imperial University, OtTo LAPORTE. Kyoto, May 6. 